\title{
EDITORIAL
}

\section{Glass half empty or half full? The story of high-flow nasal cannula therapy in critically ill children}

\author{
Padmanabhan Ramnarayan ${ }^{1 *}$ and Andreas Schibler ${ }^{2}$
}

(c) 2017 Springer-Verlag Berlin Heidelberg and ESICM

\begin{abstract}
"The optimist sees the rose and not its thorns; the pessimist stares at the thorns, oblivious of the rose"-Kahlil Gibran
\end{abstract}

High-flow nasal cannula therapy (HFNC) is the administration of heated (to body temperature) and humidified (to $>99 \%$ relative humidity) oxygen/air mixtures at flow rates that match or exceed the patient's inspiratory flow rate [1]. HFNC is well established as a mode of noninvasive respiratory support in neonatal practice $[2,3]$. Over the past decade, HFNC has also gained popularity in the management of critically ill infants and children with respiratory failure from a diverse range of diagnoses such as bronchiolitis, asthma, pneumonia, cardiac failure, neuromuscular weakness and recurrent apnoea [4]. Through various mechanisms, including washout of nasopharyngeal dead space and improved alveolar ventilation, reduction in inspiratory resistance, improvement in conductance and pulmonary compliance from heating and humidification of gases, and lung recruitment from positive distending pressure, HFNC appears to reduce the work of breathing and improve the efficiency of ventilation in children [5]. In (Fig. 1) physiological studies demonstrate that HFNC is associated with improvement in respiratory rate, heart rate, end-tidal carbon dioxide and measured work of breathing within hours of initiation, and observational studies have shown that HFNC may reduce the need for intubation and invasive ventilation [6, 7]. HFNC is also associated with high levels of patient comfort, and has the advantage of being easy to

\footnotetext{
${ }^{*}$ Correspondence: p.ramnarayan@gosh.nhs.uk

1 Children's Acute Transport Service (CATS), Great Ormond Street Hospita NHS Foundation Trust, 26-27 Boswell Street, London WC1N 3JZ, UK Full author information is available at the end of the article
}

use with relatively little staff training, including outside the intensive care unit and during inter-hospital transport $[8,9]$. Yet, niggling concerns regarding its risks (air leak, nasal trauma and abdominal distension) remain, and despite the enthusiasm for its use, no large RCTs have yet provided the much-needed evidence of its effectiveness in critically ill children.

The multicentre RCT reported by Milesi and colleagues in a recent article in Intensive Care Medicine is therefore a welcome addition to the literature [10]. The TRAMONTANE study was performed at five French paediatric intensive care units (PICU) and randomised 142 infants aged $<6$ months with moderate/severe bronchiolitis to either HFNC or nasal continuous positive airway pressure (CPAP). The primary outcome was 'treatment failure' within $24 \mathrm{~h}$ of randomisation, defined by one or more of: increase in a modified asthma score, increase in respiratory rate, increase in discomfort score and occurrence of apnoea. The study was designed as a non-inferiority trial with the accepted margin set at $15 \%$ and a power of $80 \%$. The two groups were well matched at randomisation. Treatment failure occurred in $31 \%$ of infants in the CPAP group and $50.7 \%$ of infants in the HFNC group. However, since the investigators allowed crossover in the event of treatment failure, the rate of intubation in both arms was small (3/71 infants randomised to CPAP and 5/71 infants randomised to HFNC, $\mathrm{p}=0.72$ ). In fact, 18/22 infants who 'failed' CPAP were rescued by HFNC ( $82 \%)$, while $26 / 36$ infants who 'failed' HFNC were rescued by CPAP (72\%).

The TRAMONTANE study has several strengths. The investigators recruited a relatively homogeneous population of infants with bronchiolitis, with a high enrolment rate of eligible patients $(142 / 183,78 \%)$. The entry criteria

\section{实


included an objective score used in previous bronchiolitis trials [11], and the score cut-off ensured that only infants with moderate/severe bronchiolitis were recruited. The study protocol allowed crossover if treatment failure occurred; this approach reflects real-life use of the two therapies, helping with clinical 'buy-in' and the dilemma

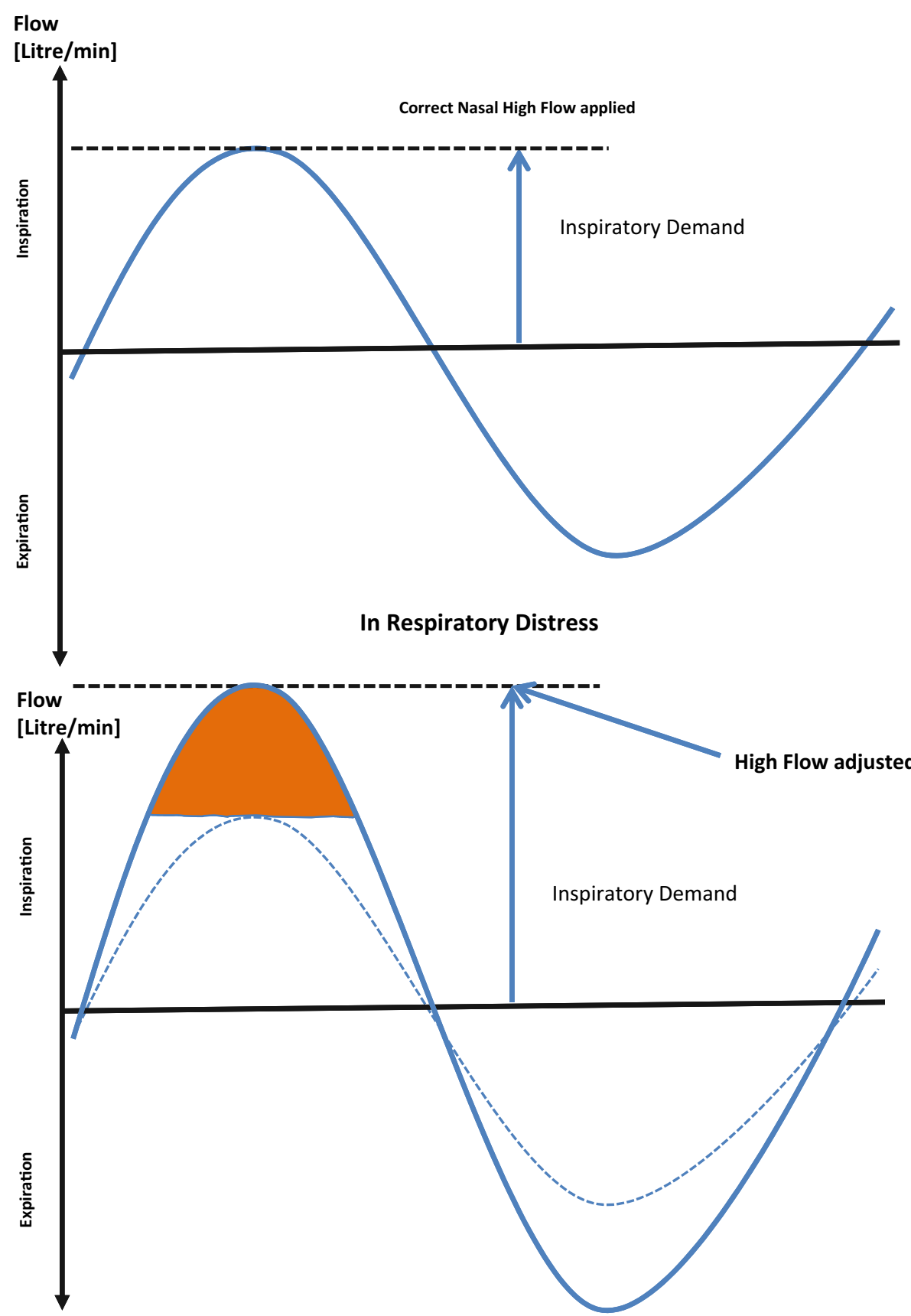

Fig. 1 The physiological effects of high flow are based on the $\mathrm{CO}_{2}$ washout effect of the nasopharyngeal dead space and on the support of the inspiratory and expiratory effort. During the expiratory phase patients are experiencing some positive airway pressures whilst exhaling against the flow into the nasopharynx. This PEEP effect is commonly described in the range of $4-6 \mathrm{cmH}_{2} \mathrm{O}$. During the inspiratory phase the important physiological effect of high flow is based on the matching of the inspiratory demand of the patient with the delivered flow rate. If the correct flow rate is applied, (upper panel) patients are experiencing a facilitated inspiration (inspiratory aid). If the inspiratory demand increases such as during respiratory distress, (lower panel) the flow rate applied needs to be increased to prevent room air entrainment around the nasal prongs and hence reducing the inspired oxygen fraction $\left(\mathrm{FiO}_{2}\right)$ 
of tolerance of each individual intervention. This trial demonstrates that HFNC and CPAP appear to be therapies that can be used interchangeably; clinician preference and experience may be the main driver for choosing one over the other.

Yet, closer examination of the trial reveals some weaknesses. First, the primary outcome is a composite endpoint comprising criteria for lack of clinical improvement as well as intolerance to the applied intervention, making it difficult to reproduce in other studies and to compare with other trials. It is also arguable whether the primary outcome is really a clinically important outcome; endotracheal intubation would certainly have been one, but the low rate of intubation in bronchiolitis in the current era $(<5 \%)$ would have necessitated a much larger sample size $[7,12]$. The duration of non-invasive ventilation (HFNC, CPAP and/or BIPAP) was greater in HFNC patients (98.3 vs $72.9 \mathrm{~h}, \mathrm{p}=0.2$ ), suggesting that length of ventilation may be a candidate outcome in future trials. Second, some may argue that the non-inferiority limit set (15\%) is generous: would clinicians really accept a failure rate of HFNC as much as $15 \%$ higher than CPAP, even if HFNC does have several benefits such as improved patient tolerance and easier nursing care? Third, although the lack of a third 'control' group of infants receiving standard low-flow oxygen could be considered problematic, recent studies suggest that the standard of care for infants with moderate/severe respiratory failure may already have shifted from low-flow oxygen to non-invasive respiratory support therapies. Recent RCTs in adult critical care also demonstrate worse outcomes for patients managed with standard oxygen therapy than for those managed with non-invasive ventilation (HFNC, CPAP or BIPAP) [13]. Fourth, the primary outcome included 'treatment failure' only within $24 \mathrm{~h}$. Only two of the infants (both randomised to HFNC) were intubated within the first $24 \mathrm{~h}$, while a further six required intubation after $24 \mathrm{~h}$, suggesting that a longer period to study 'treatment failure' may have been more appropriate.

Nevertheless, the TRAMONTANE trial addresses a key research question regarding non-invasive respiratory support in critically ill children and will hopefully pave the way for further RCTs in this area. Some clinical trials are already underway: the PARIS trial, a multicentre RCT in Australia and New Zealand comparing low-flow oxygen with HFNC in 1400 infants with bronchiolitis, has recently finished recruitment, while the FIRST ABC feasibility trial, a multicentre RCT in London comparing CPAP with HFNC as first-line non-invasive respiratory support modality in the PICU setting, has completed the target recruitment of 120 patients $[14,15]$. Results from these trials, and others to follow, will be invaluable in advancing our current state of knowledge regarding the clinical efficacy and cost effectiveness of HFNC. Encouragingly, the number of studies indexed in the National Library of Medicine using the search term "humidified high flow" or "nasal high flow" has risen from 4 in 2007 to 42 in 2016-perhaps the glass is gradually filling up after all.

\section{Author details \\ ${ }^{1}$ Children's Acute Transport Service (CATS), Great Ormond Street Hospital NHS Foundation Trust, 26-27 Boswell Street, London WC1N 3JZ, UK. ${ }^{2}$ Paediatric Intensive Care Unit, Paediatric Critical Care Research Group, Mater Research University of Queensland, Lady Cilento Children's Hospital and the University of Queensland, 501 Stanley Street, South Brisbane, QLD 4101, Australia.}

Received: 2 December 2016 Accepted: 21 December 2016

Published online: 26 January 2017

\section{References}

1. Lee JH, Rehder KJ, Williford L, Cheifetz IM, Turner DA (2013) Use of high flow nasal cannula in critically ill infants, children, and adults: a critical review of the literature. Intensive Care Med 39:247-257

2. Wilkinson D, Andersen C, O'Donnell CP, De Paoli AG, Manley BJ (2016) High flow nasal cannula for respiratory support in preterm infants. Cochrane Database Syst Rev 2:CD006405

3. Manley BJ, Owen LS, Doyle LW, Andersen CC, Cartwright DW, Pritchard MA, Donath SM, Davis PG (2013) High-flow nasal cannulae in very preterm infants after extubation. N Engl J Med 369:1425-1433

4. Hutchings FA, Hilliard TN, Davis PJ (2015) Heated humidified high-flow nasal cannula therapy in children. Arch Dis Child 100:571-575

5. Dysart K, Miller TL, Wolfson MR, Shaffer TH (2009) Research in high flow therapy: mechanisms of action. Respir Med 103:1400-1405

6. Pham TM, O'Malley L, Mayfield S, Martin S, Schibler A (2015) The effect of high flow nasal cannula therapy on the work of breathing in infants with bronchiolitis. Pediatr Pulmonol 50:713-720

7. Schibler A, Pham TM, Dunster KR, Foster K, Barlow A, Gibbons K, Hough $J \mathrm{~L}$ (2011) Reduced intubation rates for infants after introduction of highflow nasal prong oxygen delivery. Intensive Care Med 37:847-852

8. Schlapbach LJ, Schaefer J, Brady AM, Mayfield S, Schibler A (2014) Highflow nasal cannula (HFNC) support in interhospital transport of critically ill children. Intensive Care Med 40:592-599

9. Mayfield S, Bogossian F, O'Malley L, Schibler A (2014) High-Flow nasal cannula oxygen therapy for infants with bronchiolitis: pilot study. J Paediatr Child Health 50:373-378

10. Milési Christophe, Essouri Sandrine, Pouyau Robin, Liet Jean-Michel, Afanetti Mickael, Portefaix Aurélie, Baleine Julien, Durand Sabine, Combes Clémentine, Douillard Aymeric, Cambonie Gilles, Groupe Francophone de Réanimation et d'Urgences Pédiatriques (GFRUP) (2016) High flow nasal cannula (HFNC) versus nasal continuous positive airway pressure (nCPAP) for the initial respiratory management of acute viral bronchiolitis in young infants: a multicenter randomized controlled trial (TRAMONTANE study). Intensive Care Med. doi:10.1007/s00134-016-4617-8

11. Liet JM, Ducruet T, Gupta V, Cambonie G (2015) Heliox inhalation therapy for bronchiolitis in infants. Cochrane Database Syst Rev 9:CD006915

12. Essouri S, Laurent M, Chevret L, Durand P, Ecochard E, Gajdos V, Devictor D, Tissieres P (2014) Improved clinical and economic outcomes in severe bronchiolitis with pre-emptive nCPAP ventilatory strategy. Intensive Care Med 40:84-91

13. Frat JP, Thille AW, Mercat A, Girault C, Ragot S, Perbet S, Prat G, Boulain T, Morawiec E, Cottereau A, Devaquet J, Nseir S, Razazi K, Mira JP, Argaud L, Chakarian JC, Ricard JD, Wittebole X, Chevalier S, Herbland A, Fartoukh 
M, Constantin JM, Tonnelier JM, Pierrot M, Mathonnet A, Beduneau G, Deletage-Metreau C, Richard JC, Brochard L, Robert R, Group FS, the RN, (2015) High-flow oxygen through nasal cannula in acute hypoxemic respiratory failure. N Engl J Med 372:2185-2196

14. Franklin D, Dalziel S, Schlapbach LJ, Babl FE, Oakley E, Craig SS, Furyk JS, Neutze J, Sinn K, Whitty JA, Gibbons K, Fraser J, Schibler A, Paris, Predict
(2015) Early high flow nasal cannula therapy in bronchiolitis, a prospective randomised control trial (protocol): a Paediatric Acute Respiratory Intervention Study (PARIS). BMC Pediatr 15:183

15. Clinical Trials website. http://clinicaltrials.gov/ct2/show/NCT02612415. Accessed 1 Dec 2016 